\title{
Approximate Distance Oracle with Constant Query Time
}

\author{
Shiri Chechik*
}

October 5, 2018

\begin{abstract}
An approximate distance oracle is a succinct data structure that provides fast answers to distance queries between any two nodes.

In this paper we consider approximate distance oracles for general undirected graphs with non-negative edge weights with constant query time. We present a distance oracle of size $O\left(k n^{1+1 / k}\right)$, with $2 k-1$ stretch and $O(1)$ query time. This improves the $O(\log k)$ query time of Wulff-Nilsen's distance oracle [SODA '13], which in turn improved the $O(k)$ query time of Thorup and Zwick's distance oracle [J. ACM '05].
\end{abstract}

\section{Introduction}

Finding shortest paths is perhaps one of the most fundamental and studied computational problems. A great number of papers deal with different variants of this problem. For example, the well known Dijkstra and Bellman-Ford algorithms allow computing the shortest path distance between any pair of nodes. In many applications it is desirable to retrieve shortest path distances extremely fast, ideally in time that is independent of the network size.

A distance oracle is a data structure that allows fast retrieval of a distance estimate for any pair of nodes. A naive solution to accomplish this is to invoke an all pairs shortest paths algorithm and store the distance matrix. Using the pre-computed distance matrix, distance queries can be answered in constant time. The main disadvantages of this solution is that the space may be too large (quadratic in the number of nodes) and that computing all pairs shortest paths may take too long. To overcome these drawbacks, much of the work on distance oracles considers approximated distances.

The distance oracle is said to be of stretch $k$ (or $k$-approximate distance oracle), if for every two vertices $s$ and $t$, the distance $\widehat{\operatorname{dist}}(s, t)$ returned by the distance oracle satisfies $\operatorname{dist}(s, t) \leq$ $\widehat{\operatorname{dist}}(s, t) \leq k \cdot \operatorname{dist}(s, t)$.

The focus on designing distance oracles is often on the tradeoff between several parameters: the construction time (the time it takes to construct the distance oracle), the size of the data structure, the query time and the stretch guarantee.

Awerbuch et al. [2] presented a distance oracle with stretch $64 k, \tilde{O}\left(k n^{1+1 / k}\right)$ size, $\tilde{O}\left(m n^{1 / k}\right)$ construction time and $O\left(k n^{1 / k}\right)$ query time. Cohen [5] improved the stretch to $2 k+\epsilon$ and later Matoušek [7] further improved the stretch to $2 k-1$ using completely different techniques.

${ }^{*}$ Microsoft Research Silicon Valley, Mountain View CA, USA. Email: schechik@microsoft.com. 
In a seminal paper Thorup and Zwick [14] significantly improved the query time, presenting a distance oracle with $2 k-1$ stretch, $O\left(k n^{1+1 / k}\right)$ expected size, $O\left(k m n^{1 / k}\right)$ construction time and $O(k)$ query time. The stretch-space tradeoff is essentially optimal up to the $k$ factor in the space, assuming the girth conjecture of Erdős [6]. Roditty, Thorup, and Zwick [13] later show how to de-randomize the construction while keeping the same bounds. Baswana and Kavitha [4] presented an improved construction time for dense graphs of $O\left(n^{2} \log n\right)$ with query time of $O(k)$ for $k>2$ and of $\Theta(\log n)$ for $k=2$.

Wulff-Nilsen [15] presented a distance oracle with subquadratic time when $m=o\left(n^{2}\right)$, presenting a distance oracle with $2 k-1$ stretch, $O\left(k n^{1+1 / k}\right)$ size, $O(k)$ query time and $O\left(\sqrt{k} m+k n^{1+c / \sqrt{k}}\right)$ construction time for some absolute constant $c$.

Pătraşcu and Roditty [12] presented a distance oracle for unweighted graphs of size $O\left(n^{5 / 3}\right)$ with a multiplicative stretch 2 and additive stretch 1 . In addition, they present a distance oracle for weighted graphs of size $O\left(n^{2} / \sqrt[3]{\alpha}\right)$ where $\alpha=n^{2} / m$.

Later Abraham and Gavoille [1] presented a distance oracle of size $\tilde{O}\left(n^{1+2 /(2 k-1)}\right)$ with $O(k)$ query time and with a multiplicative stretch $2 k-2$ and additive stretch 1 .

Baswana et al. [3] also considered distance oracles with both additive and multiplicative stretch, presenting a distance oracle of size $O\left(k n^{1+1 / k}\right)$, with a multiplicative stretch $2 k-1$ and additive stretch 2 with subquadratic construction time of $O\left(\min \left(m+k n^{3 / 2+1 /(2 k)+1 /(2 k-2)}, k m n^{1 / k}\right)\right)$.

Mendel and Naor [8, 9] studied approximate distance oracles with constant query time. They presented an approximate distance oracle with size $O\left(n^{1+1 / k}\right), 128 k$ stretch, $O(1)$ query time and $O\left(n^{2+1 / k} \log n\right)$ construction time. The $128 k$ stretch can be improved to $33 k$ using techniques of Naor and Tao [11, and according to Naor and Tao the stretch can be further improved to $16 k$ using a more careful analysis. In addition, Mendel and Schwob [10] improved the $O\left(n^{2+1 / k} \log n\right)$ construction time to $O\left(m n^{1 / k} \log ^{3} n\right)$.

Wulff-Nilsen [16] improved the $128 k$ stretch of Manor and Naor's construction to $(2 k+\epsilon)$ at the cost of additional $k$-factor in the size, the query time of his construction is $O(1 / \epsilon)$ and the construction time is $O\left(k m n^{1 / k}+k n^{1+1 / k} \log n+m n 1 /(c k) \log ^{3} n\right)$. For the case of $k=O(\log n / \log \log n)$ and a fixed $\epsilon$, Wulff-Nilsen showed that it is actually possible to reduce the size back to $O\left(n^{1+1 / k}\right)$. In addition, Wulff-Nilsen [16] showed that it is possible to improve the query time of Thorup and Zwick's distance oracle [14] from $O(k)$ to $O(\log k)$. Namely, a distance oracle of size $O\left(k n^{1+1 / k}\right)$, $2 k-1$ stretch, $O(\log k)$ query time.

In this paper we further improve the $O(\log k)$ query time of Wulff-Nilsen's distance oracle [16] from $O(\log k)$ to a universal constant. More precisely, we show a distance of size $O\left(k n^{1+1 / k}\right), 2 k-1$ stretch, $O(1)$ query time and $O\left(k m n^{1 / k}+k n^{1+1 / k} \log n+m n 1 /(c k) \log ^{3} n\right)$ construction time.

Our algorithm first invokes the Mendel and Naor [9] distance oracle to obtain an initial distance estimation. We then show that using this distance estimation it is possible to get $2 k-1$ stretch in constant time using the Thorup-Zwick distance oracle and some additional information.

\section{$1.1 \quad$ Preliminaries}

For completeness we first outline the construction of Thorup-Zwick distance oracle [14.

For a given positive integer $k$, the sets $V=A_{0} \supseteq A_{1} \supseteq \cdots \supseteq A_{k-1}$ are constructed as follows. Set $A_{0} \leftarrow V$ and $A_{k} \leftarrow \emptyset$. The set $A_{i}$ for $1<i \leq k-1$ is obtained by sampling the vertices of $A_{i-1}$ independently at random with probability $n^{-1 / k}$.

For pair of nodes $u$ and $v$, let $\operatorname{dist}(u, v)$ be their distance in $G$. The pivot $p_{i}(v)$ is defined to be the closest node to $v$ in $A_{i}$ (break ties arbitrarily). 
The bunch of $v$ is defined as follows,

$$
B(v)=\bigcup_{i=0}^{k-1} B_{i}(v),
$$

where

$$
B_{i}(v)=\left\{u \in A_{i} \backslash A_{i+1} \mid \operatorname{dist}(v, u)<\operatorname{dist}\left(v, p_{i+1}(v)\right)\right\} .
$$

The query algorithm given pair of nodes $s$ and $t$ is done as follows. Let $w \leftarrow s, j \leftarrow 0$. While $w \notin B(t)$ do the following. Set $j \leftarrow j+1,(s, t) \leftarrow(t, s)$ and $w \leftarrow p_{j}(s)$. In the end of the while loop return $\operatorname{dist}(s, w)+\operatorname{dist}(w, t)$.

It was shown in [14 that the expected size of each bunch is $O\left(k \cdot n^{1 / k}\right)$ and that all bunches can be constructed in $O\left(k m n^{1 / k}\right)$ time.

\section{Oracle with Constant Query Time}

Let us start with some notations. Consider the sets $A_{0}, \ldots, A_{k}$ from Thorup-Zwick distance oracle [14. Let $\delta_{j}(u)=\operatorname{dist}\left(u, p_{j}(u)\right)-\operatorname{dist}\left(u, p_{j-2}(u)\right)$. Let $\Delta_{j}(u)=\max _{1 \leq i \leq j, i \text { is even }} \delta_{i}(u)$.

Let $I(i, u)$ be the even index such that $I(i, u) \leq i$ and $\delta_{I(i, u)}(u)$ is maximal, i.e., $\delta_{I(i, u)}(u)=\Delta_{i}(u)$

Definition 1 (Legitimate Pair) We say that two indices $i_{1}$ and $i_{2}$ are legitimate pair for the pair $s, t$ if the following holds:

1. $i_{2}$ and $i_{1}$ are even.

2. $\Delta_{i_{1}}(s) / 2 \leq \operatorname{dist}(s, t)$.

3. $p_{I\left(i_{2}-2, s\right)}(s) \in B(t)$ or $p_{I\left(i_{2}-1, s\right)}(t) \in B(s)$.

4. $\operatorname{dist}\left(s, p_{i_{2}}\right) \leq 2 \operatorname{dist}\left(s, p_{i_{1}}\right)$.

Our algorithm consists of two parts. The first part finds legitimate indices $i_{1}$ and $i_{2}$, the second part uses these indices to get $2 k-1$ stretch. In Section 2.1] we describe the preprocessing phase and the query phase for finding legitimate pair for a given pair of vertices $s$ and $t$. In Section 2.2 we describe the preprocessing phase and the query phase for estimating the distance within stretch $2 k-1$ given pair of nodes $s$ and $t$ and legitimate pair $i_{1}$ and $i_{2}$.

\subsection{Finding Legitimate Pair}

We now turn to describe the preprocessing and query phases of the first part of the algorithm, which finds legitimate pair of indices given pair of nodes $s$ and $t$. In order to find a legitimate indices $i_{1}$ and $i_{2}$ we use the techniques introduced in [16] and adapt them to our needs.

Let us start with describing the information stored at the preprocessing phase.

Construct the Thorup and Zwick distance oracle. Let $\mathcal{D}_{T Z}=\{\operatorname{dist}(u, v) \mid u, v \in V$ and $u \in$ $B(v)\} \cup\left\{\boldsymbol{d i s t}\left(u, p_{i}(u)\right) \mid u \in V, 1 \leq i \leq k-1\right\}$. For every node $v$ and index $1 \leq j \leq k-1$, store $\Delta_{j}(u)$ and the index $I(j, u)$.

Construct the Mendel and Naor [9] distance oracle. Let $\operatorname{dist}_{M N}(u, v)$ be the estimated distance returned by the Mendel-Naor distance oracle for the pair $u$ and $v$. Let $\mathcal{D}_{M N}=\left\{\boldsymbol{d i s t}_{M N}(u, v) \mid\right.$ $u, v \in V\}$ be the set of all distances that the Mendel-Naor distance oracle can output. 
Let $\mathcal{D}=\mathcal{D}_{M N} \cup \mathcal{D}_{T Z}$.

Let $\tilde{\mathcal{S}}$ be the set obtained by the following process. Initially set $\tilde{\mathcal{S}} \leftarrow \emptyset$. Consider the elements of $\mathcal{D}$ in a decreasing order, add the current element $x$ to $\tilde{\mathcal{S}}$ if the previous added element is greater than $2 x$. Let $\tilde{\mathcal{D}}$ be an array containing all elements of $\tilde{\mathcal{S}}$ in an increasing order. In addition store in a hash $\tilde{\mathcal{H}}$ all elements $\tilde{d}$ in $\tilde{\mathcal{D}}$, where $\tilde{d}$ is the key and the value is the index $j$ such that $\tilde{\mathcal{D}}[j]=\tilde{d}$.

It is not hard to verify that the set $\tilde{\mathcal{D}}$ satisfies the following properties. First, for every element $d \in \mathcal{D}$ there is an element $\tilde{d}$ in $\tilde{\mathcal{D}}$ such that $d \leq \tilde{d} \leq 2 d$. Second, every two consecutive elements $\tilde{d}_{1}$ and $\tilde{d}_{2}$ in $\tilde{\mathcal{D}}$ such that $\tilde{d}_{1}<\tilde{d}_{2}$ satisfy $\tilde{d}_{1} \leq \tilde{d}_{2} / 2$.

For every distance $d \in \mathcal{D}$ store (in a hash) a pointer to the distance $\tilde{\mathcal{D}}(d) \in \tilde{\mathcal{D}}$ such that $\tilde{\mathcal{D}}(d)$ is the minimal value in $\tilde{\mathcal{D}}$ such that $d \leq \tilde{\mathcal{D}}(d)$. For a value $d^{\prime}$ such that $d^{\prime} \notin \mathcal{D}, \tilde{\mathcal{D}}\left(d^{\prime}\right)$ is undefined.

For every node $u$ and index $i$, let $\tilde{d}(i, u)$ be the minimal element in $\tilde{\mathcal{D}}$ such that $\operatorname{dist}\left(u, p_{i}(u)\right) \leq$ $\tilde{d}(i, u)$. For every node $u$, store the distances $\tilde{d}(i, u)$ in a hash $H_{u}$, where the key is $\tilde{d}(i, u)$ and the value is $i$. In addition store the values $\tilde{d}(i, u)$ in a sorted array $L_{u}$ in an increasing order. Store pointers from every index $1 \leq i \leq k-1$ to the element $\tilde{d}(i, u)$ in $L_{u}$.

Let $\tilde{\mathcal{D}}[\tilde{d}, i]$ be the $i$ 'th element in $\tilde{\mathcal{D}}$ after $\tilde{d}$ or undefined in case $\tilde{d}$ is not in $\tilde{\mathcal{D}}$. Namely, let $j$ be the index such that $\tilde{\mathcal{D}}[j]=\tilde{d}$, then $\tilde{\mathcal{D}}[\tilde{d}, i]=\tilde{\mathcal{D}}[j+i]$. If $j+i<0$ then return the first element of $\tilde{\mathcal{D}}$. Similarly, if $\tilde{\mathcal{D}}$ contains less than $j+i$ elements return the last one.

Similarly, we define $L_{u}[\tilde{d}, i]$, namely, $L_{u}[d, i]$ is the $i$ 'th element in $L_{u}$ after $\tilde{d}$ or undefined in case $\tilde{d}$ is not in $L_{u}$. Namely, let $j$ be the index such that $L_{u}[j]=\tilde{d}$, then $L_{u}[\tilde{d}, i]=\tilde{\mathcal{D}}[j+i]$. If $j+i<0$ then return the first element in $L_{u}$. Similarly, if $L_{u}$ contains less than $j+i$ elements return the last one.

Note that $\tilde{\mathcal{D}}[\tilde{d}, i]$ (and similarly $L_{u}[\tilde{d}, i]$ ) can be accesses in $O(1)$ time. This can be done as follows. Recall that for every distance $\tilde{d}$ that belongs to $\tilde{\mathcal{D}}$ the algorithm stores (in a hash) the index $j$ such that $\tilde{\mathcal{D}}[j]=\tilde{d}$. Hence the index $j$ can be retrieved in constant time. It is not hard to see now that the value $\tilde{\mathcal{D}}[\tilde{d}, i]=\tilde{\mathcal{D}}[j+i]$ can be retrieved in constant time.

For every $\tilde{d} \in L_{u}$ and node $u$, let $\overline{\operatorname{even}}_{u}(\tilde{d})$ be the even index $i$ with maximal $\operatorname{dist}\left(u, p_{i}(u)\right)$ such that $\tilde{\mathcal{D}}\left(\operatorname{dist}\left(u, p_{i}(u)\right)\right)=\tilde{d}$ and let $\underline{\text { even }}_{u}(\tilde{d})$ be the minimal.

We now turn describing how to find legitimate indices in the query phase.

Let us first describe a procedure that will be used several times in the query algorithm. Procedure Check-Ind $(s, t, i)$ gets an even index $i$ and nodes $s$ and $t$ and returns a distance $\hat{d}$ that satisfies the following. 1. Either $\hat{d}=-1, i<k-2$, and $\operatorname{dist}(s, t) \geq \Delta_{i}(s) / 2$, or 2 . $\operatorname{dist}(s, t) \leq \hat{d} \leq(2 k-1) \operatorname{dist}(s, t)$, or 3 . $\operatorname{dist}(s, t) \leq \hat{d} \leq 2 \operatorname{dist}\left(s, p_{i-2}(s)\right)+3 \operatorname{dist}(s, t)$.

Procedure Check-Ind $(s, t, i)$ operates as follows. Let $j=I(s, i)$. If $p_{j-2}(s) \in B(t)$ return $\operatorname{dist}\left(s, p_{j-2}(s)\right)+\operatorname{dist}\left(t, p_{j-2}(s)\right)$. Else if $p_{j-1}(t) \in B(s)$ return $\operatorname{dist}\left(s, p_{j-1}(t)\right)+\operatorname{dist}\left(t, p_{j-1}(t)\right)$. Else if $i=k-2$ then if $p_{i}(s) \in B(t)$ return $\operatorname{dist}\left(s, p_{i}(s)\right)+\operatorname{dist}\left(t, p_{i}(s)\right)$, else return $\operatorname{dist}\left(s, p_{k-1}(t)\right)+$ $\operatorname{dist}\left(t, p_{k-1}(t)\right)$. Else if $i=k-1$ then return $\operatorname{dist}\left(s, p_{k-1}(s)\right)+\operatorname{dist}\left(t, p_{k-1}(s)\right)$. Else return -1 .

To find a legitimate pair $i_{1}, i_{2}$ in the query algorithm for given nodes $s$ and $t$ do the following. First invoke the Mendel and Naor [9] distance oracle to obtain an initial estimation $\operatorname{dist}_{M N}(s, t)$. $\operatorname{Recall~that~} \operatorname{dist}(s, t) \leq \operatorname{dist}_{M N}(s, t) \leq 128 k \operatorname{dist}(s, t)$. Let $\tilde{d}=\tilde{\mathcal{D}}\left(\operatorname{dist}_{M N}(s, t)\right)$. The simplest case is when either $s \in B(t)$ or $t \in B(s)$. In this case the algorithm can extract the exact distance $\operatorname{dist}(s, t)$ in constant time. In this case the algorithm returns $(-1,-1, \operatorname{dist}(s, t))$.

Otherwise, an attempt is made to find the maximal value $\tilde{d}_{\text {min }}$ in $L_{s}$ (or $L_{t}$ ) such that $\tilde{d}_{\text {min }} \leq$ $\operatorname{dist}_{M N}(s, t) / 256$.

This is done as follows. Set $i=2$ and found $1 \leftarrow$ false. While $(i>-9)$ and ( found $1=$ false) do the following. Let $\tilde{d}_{\text {curr }}=\tilde{\mathcal{D}}[\tilde{d}, i]$. Check if $H_{s}\left(\tilde{d}_{\text {curr }}\right) \neq$ null or $H_{t}\left(\tilde{d}_{\text {curr }}\right) \neq$ null then 
found $1=$ true. Else $i=i-1$. By the end of the while loop, check if ( found $1=$ false), if so return $\left(-1,-1,2 \tilde{d}_{\text {curr }}\right)$. Otherwise assume w.l.o.g. that $H_{s}\left(\tilde{d}_{c u r r}\right) \neq$ null (otherwise switch $s$ and $t$ ). Move down the list $L_{s}$ from the value $\tilde{d}_{c u r r}$ until finding a value $\tilde{d}_{\text {min }}$ such that $\tilde{d}_{\text {min }} \leq \operatorname{dist}_{M N}(s, t) / 256$. Let $i_{\text {min }} \leftarrow \overline{\operatorname{even}}_{s}\left(\tilde{d}_{\text {min }}\right)$. Let $\ell=0$. Move up the list $L_{s}$ until finding a value $L_{s}\left[\tilde{d}_{m i n}, \ell\right]$ such that Check-Ind $(s, t, i) \neq-1$ where $i=\overline{e v e n}_{s}\left(L_{s}\left[\tilde{d}_{\text {min }}, \ell\right]\right)$. This is done until either finding such a value $L_{s}\left[\tilde{d}_{\text {min }}, \ell\right]$ or until $L_{s}\left[\tilde{d}_{\text {min }}, \ell-2\right]>\tilde{d}$. If no such value found return $\left(-1,-1, \operatorname{dist}_{M N}(s, t)\right)$. Else let $i_{2} \leftarrow I\left(s, \overline{\text { even }}_{s}\left(L_{s}\left[\tilde{d}_{\text {min }}, \ell\right]\right)\right)$ and $i_{1} \leftarrow$ even $_{s}\left(L_{s}\left[\tilde{d}_{\text {min }}, \ell\right]\right)$. If Check-Ind $\left(s, t, i_{1}\right) \neq-1$, then return $\left(-1,-1\right.$, Check-Ind $\left.\left(s, t, i_{1}\right)\right)$. Else return $\left(i_{1}, i_{2},-1\right)$.

See Procedure Find-Legitimate for the pseudo-code.

Let us now turn to the analysis of the Procedure Find-Legitimate.

We start by an auxiliary claim.

Claim 2.1 Consider an even index $i$ such that $2 \leq i \leq k-1$, one of the following happens: either 1. $\operatorname{dist}(s, t) \leq \delta_{i}(s) / 2$ or 2. $p_{i-2}(s) \in B(t)$ or 3. $p_{i-1}(t) \in B(s)$.

Proof: If either $p_{i-2}(s) \in B(t)$ or $p_{i-1}(t) \in B(s)$ we are done, so assume $p_{i-2}(s) \notin B(t)$ and $p_{i-1}(s) \notin B(s)$.

Note that by definition of $B(s)$ and $B(t)$ we have $\operatorname{dist}\left(s, p_{i}(s)\right) \leq \operatorname{dist}\left(s, p_{i-1}(t)\right)$ and $\operatorname{dist}\left(t, p_{i-1}(t)\right) \leq$ $\operatorname{dist}\left(t, p_{i-2}(s)\right)$.

$\operatorname{dist}\left(s, p_{i}(s)\right) \leq \operatorname{dist}\left(s, p_{i-1}(t)\right) \leq \operatorname{dist}(s, t)+\operatorname{dist}\left(t, p_{i-1}(t)\right) \leq \operatorname{dist}(s, t)+\operatorname{dist}\left(t, p_{i-2}(s)\right) \leq$ $2 \operatorname{dist}(s, t)+\operatorname{dist}\left(s, p_{i-2}(s)\right)$. We get that $\operatorname{dist}\left(s, p_{i}(s)\right)-\operatorname{dist}\left(s, p_{i-2}(s)\right) \leq 2 \operatorname{dist}(s, t)$. Hence $\operatorname{dist}(s, t) \geq \delta_{i}(s) / 2$, as required.

We now turn to the correctness of Procedure Check-Ind.

Lemma 2.1 Procedure Check-Ind $(s, t, i)$ gets an even index $i$ and nodes $s$ and $t$ and returns a distance $\hat{d}$ that satisfies the following. 1. Either $\hat{d}=-1, i<k-2$, and $\operatorname{dist}(s, t) \geq \Delta_{i}(s) / 2$, or 2 . $\operatorname{dist}(s, t) \leq \hat{d} \leq(2 k-1) \operatorname{dist}(s, t)$, or 3 . $\operatorname{dist}(s, t) \leq \hat{d} \leq 2 \operatorname{dist}\left(s, p_{i-2}(s)\right)+3 \operatorname{dist}(s, t)$.

Proof: Let $j=I(s, i)$.

If $p_{j-2}(s) \in B(t)$ then the Procedure returns $\operatorname{dist}\left(s, p_{j-2}(s)\right)+\operatorname{dist}\left(t, p_{j-2}(s)\right)$. Note that $\operatorname{dist}\left(s, p_{j-2}(s)\right)+\operatorname{dist}\left(t, p_{j-2}(s)\right) \leq \operatorname{dist}\left(s, p_{j-2}(s)\right)+\operatorname{dist}\left(s, p_{j-2}(s)\right)+\operatorname{dist}(s, t)=2 \operatorname{dist}\left(s, p_{j-2}(s)\right)+$ $\operatorname{dist}(s, t)<2 \operatorname{dist}\left(s, p_{j-2}(s)\right)+3 \operatorname{dist}(s, t) \leq 2 \operatorname{dist}\left(s, p_{i-2}(s)\right)+3 \operatorname{dist}(s, t)$.

Consider now the case where $p_{j-2}(s) \notin B(t)$ and $p_{j-1}(t) \in B(s)$. In this case the algorithm returns $\operatorname{dist}\left(s, p_{j-1}(t)\right)+\operatorname{dist}\left(t, p_{j-1}(t)\right)$. Note that since $p_{j-2}(s) \notin B(t)$, we have $\operatorname{dist}\left(t, p_{j-1}(t)\right) \leq$ $\operatorname{dist}\left(t, p_{j-2}(s)\right) \leq \operatorname{dist}\left(s, p_{j-2}(s)\right)+\operatorname{dist}(s, t)$. Hence, $\operatorname{dist}\left(s, p_{j-1}(t)\right)+\operatorname{dist}\left(t, p_{j-1}(t)\right) \leq 2 \operatorname{dist}\left(t, p_{j-1}(t)\right)+$ $\operatorname{dist}(s, t) \leq 2 \operatorname{dist}\left(s, p_{j-2}(s)\right)+2 \operatorname{dist}(s, t)+\operatorname{dist}(s, t)=2 \operatorname{dist}\left(s, p_{j-2}(s)\right)+3 \operatorname{dist}(s, t) \leq 2 \operatorname{dist}\left(s, p_{i-2}(s)\right)+$ $3 \operatorname{dist}(s, t)$.

Assume $p_{j-2}(s) \notin B(t)$ and $p_{j-1}(t) \notin B(s)$. In this case we have $\operatorname{dist}\left(s, p_{j}(s)\right) \leq \operatorname{dist}\left(s, p_{j-1}(t)\right) \leq$ $\operatorname{dist}(s, t)+\operatorname{dist}\left(t, p_{j-1}(t)\right) \leq \operatorname{dist}(s, t)+\operatorname{dist}\left(t, p_{j-2}(t)\right) \leq 2 \operatorname{dist}(s, t)+\operatorname{dist}\left(s, p_{j-2}(t)\right)$. We get that $\operatorname{dist}\left(s, p_{j}(s)\right)-\operatorname{dist}\left(s, p_{j-2}(t)\right) \leq 2 \operatorname{dist}(s, t)$. Hence $\operatorname{dist}(s, t) \geq \Delta_{i}(s) / 2$.

Consider now the case where $i=k-2$ and $p_{i}(s) \in B(t)$, in this case the algorithm returns $\operatorname{dist}\left(s, p_{i}(s)\right)+\operatorname{dist}\left(t, p_{i}(s)\right)$. Note that $\operatorname{dist}\left(s, p_{i}(s)\right) \leq i \Delta_{i}(s) / 2$. We get that $\operatorname{dist}\left(s, p_{i}(s)\right)+$ $\operatorname{dist}\left(t, p_{i}(s)\right) \leq 2 \operatorname{dist}\left(s, p_{i}(s)\right)+\operatorname{dist}(s, t) \leq 2 i \Delta_{i}(s) / 2+\operatorname{dist}(s, t) \leq 2(k-2) \Delta_{i}(s) / 2+\operatorname{dist}(s, t) \leq$ $(2 k-3) \operatorname{dist}(s, t)$.

Consider the case where $i=k-2, p_{i}(s) \notin B(t)$ and the algorithm returns $\operatorname{dist}\left(s, p_{k-1}(t)\right)+$ $\operatorname{dist}\left(t, p_{k-1}(t)\right)$. We get that $\operatorname{dist}\left(s, p_{k-1}(t)\right)+\operatorname{dist}\left(t, p_{k-1}(t)\right) \leq 2 \operatorname{dist}\left(t, p_{k-1}(t)\right)+\operatorname{dist}(s, t) \leq$ $2 \operatorname{dist}\left(t, p_{k-2}(s)\right)+\operatorname{dist}(s, t) \leq(2 k-1) \operatorname{dist}(s, t)$. 
The case where $i=k-1$ and the algorithm returns $\operatorname{dist}\left(s, p_{k-1}(s)\right)+\operatorname{dist}\left(t, p_{k-1}(s)\right)$ can be proved similarly to the previous case.

Finally, consider the last case where the algorithm returns -1 . Note that in this case $\operatorname{dist}(s, t) \geq$ $\Delta_{i}(s) / 2$ and $i<k-2$ as required.

We are now ready to prove the correctness of Procedure Find-Legitimate.

Lemma 2.2 Let $\left(i_{1}, i_{2}, \hat{d}\right)$ be the tuple returned by Procedure Find-Legitimate, then either $\hat{d}=-1$ and $\left(i_{1}, i_{2}\right)$ is a legitimate pair for $s$ and $t$ or $\operatorname{dist}(s, t) \leq \hat{d} \leq(2 k-1) \operatorname{dist}(s, t)$.

Proof: Note that the algorithm returns a value in one of the lines: $2,5,11,14,17,18$. We show that the value returned in each such line satisfies the lemma.

Consider the case where the algorithm returns a value in line 2. Note that if $s \in B(t)$ or $t \in B(s)$ then the exact distance $\operatorname{dist}(s, t)$ can be extracted in $O(1)$ time.

Consider the case where the algorithm returns a value in line 5 . Let $\left(-1,-1,2 \tilde{d}_{\text {curr }}\right)$ be the tuple returned by the algorithm. The algorithm returns a value in line 5 of the algorithm in case found $1=$ false when the algorithm reaches line 5 . This happens only when none of the values $\tilde{\mathcal{D}}[\tilde{d}, i]$ for $-9 \leq i \leq 2$ exists in both $H_{s}$ and $H_{t}$. In other words, both $H_{s}$ and $H_{t}$ do not contain values between $\tilde{\mathcal{D}}[\tilde{\tilde{d}},-9]$ to $\tilde{\mathcal{D}}[\tilde{d}, 2]$. This means that both $H_{s}$ and $H_{t}$ do not contain values between $\tilde{d} / 2^{-9}$ to $4 \tilde{d}$.

We claim that $\tilde{d}_{\text {curr }} \leq \tilde{d} / 2^{-9}$. Note that the only case where $\tilde{d}_{\text {curr }}>\tilde{d} / 2^{-9}$ is when $\tilde{\mathcal{D}}$ contains less than nine elements that are smaller than $\tilde{d}$.

we claim that both $H_{s}$ and $H_{t}$ contains a value smaller or equal to $\tilde{d}$. To see this note that as $t \in B(s)$, we have $\operatorname{dist}\left(s, p_{1}(s)\right) \leq \operatorname{dist}(s, t) \leq \operatorname{dist}_{M N}(s, t)$. Hence $\tilde{\mathcal{D}}\left(\boldsymbol{d i s t}\left(s, p_{1}(s)\right)\right) \leq$ $\tilde{\mathcal{D}}\left(\operatorname{dist}_{M N}(s, t)\right)=\tilde{d}$.

Note also that $\tilde{\mathcal{D}}\left(\boldsymbol{\operatorname { d i s t }}\left(s, p_{1}(s)\right)\right)$ is contained in both $H_{s}$ and $\tilde{\mathcal{D}}$. It follows that by moving down the list of $\tilde{\mathcal{D}}$ the algorithm either encounters the value $\tilde{\mathcal{D}}\left(\operatorname{dist}\left(s, p_{1}(s)\right)\right)$ or it doesn't reach the beginning of the list. We get that $\tilde{d}_{c u r r} \leq \tilde{d} / 2^{-9}$.

Hence $\tilde{d}_{c u r r} \leq \tilde{d} / 2^{-9} \leq 2 \operatorname{dist}_{M N}(s, t) / 2^{-9}=\operatorname{dist}_{M N}(s, t) / 2^{-8} \leq 128 k \operatorname{dist}(s, t) / 2^{-8}=k \operatorname{dist}(s, t) / 2$. Hence $2 \tilde{d}_{c u r r} \leq k \operatorname{dist}(s, t)<(2 k-1) \operatorname{dist}(s, t)$. We need to show the other direction, namely, $\operatorname{dist}(s, t) \leq 2 \tilde{d}_{\text {curr }}$.

Let $j$ be the maximal even index such that $\operatorname{dist}\left(s, p_{j}(s)\right) \leq \tilde{d}_{c u r r}$. Note that such an index exists as $\operatorname{dist}\left(s, p_{0}(s)\right)=0 \leq \tilde{d}_{\text {curr }}$. Recall also that $H_{s}$ does does not contain any value in $\tilde{\mathcal{D}}[\tilde{d}, i]$ for $-9 \leq i \leq 2$.

This implies that either $j+2>k-1$ or that $\tilde{\mathcal{D}}\left(\operatorname{dist}\left(s, p_{j+2}(s)\right)\right)>\tilde{\mathcal{D}}[\tilde{d}, 2] \geq 4 \tilde{d} \geq 4 \operatorname{dist}(s, t)$.

In the latter case we have $\Delta_{j+2}(s)>2 \operatorname{dist}(s, t)$. Hence by claim 2.1, either $p_{j}(s) \in B(t)$ or $p_{j+1}(t) \in B(s)$. Note that also in the first case we have either $p_{j}(s) \in B(t)$ or $p_{j+1}(t) \in B(s)$.

If $p_{j}(s) \in B(t)$, then note that $\operatorname{dist}\left(p_{j}(s), t\right) \leq \operatorname{dist}(s, t)+\operatorname{dist}\left(s, p_{j}(s)\right)<\operatorname{dist}(s, t)+\tilde{d}_{c u r r}<$ $\tilde{\mathcal{D}}[\tilde{d}, 2]$. But recall that, $H_{t}$ does not contain values between $\tilde{\mathcal{D}}[\tilde{d},-9]$ to $\tilde{\mathcal{D}}[\tilde{d}, 2]$. It follows that $\operatorname{dist}\left(t, p_{j}(s)\right) \leq \tilde{\mathcal{D}}\left(\operatorname{dist}\left(t, p_{j}(s)\right)\right) \leq \tilde{d}_{c u r r}$. Hence $\operatorname{dist}\left(s, p_{j}(s)\right)+\operatorname{dist}\left(t, p_{j}(s)\right) \leq 2 \tilde{d}_{c u r r}$, as required.

The case where $p_{j+1}(t) \in B(s)$ is handled similarly.

Consider the case where the algorithm returns a value in line 11 . In this case, Check-Ind $\left(s, t, i_{\min }\right) \neq$ -1 . By Lemma $2.1 \hat{d}$ satisfies one of the following. Either $\operatorname{dist}(s, t) \leq \hat{d} \leq(2 k-1) \operatorname{dist}(s, t)$ or $\operatorname{dist}(s, t) \leq \hat{d} \leq 2 \operatorname{dist}\left(s, p_{i_{\text {min }}-2}(s)\right)+3 \operatorname{dist}(s, t)$. In the first case the lemma holds. Consider the second case. Note that in this case $\tilde{d}_{\text {min }} \leq \operatorname{dist}_{M N}(s, t) / 256 \leq \operatorname{dist}(s, t) / 2$. We get that $\operatorname{dist}(s, t) \leq \hat{d} \leq 2 \operatorname{dist}\left(s, p_{i_{\text {min }}-2}(s)\right)+3 \operatorname{dist}(s, t) \leq 2 \tilde{d}_{\text {min }}+3 \operatorname{dist}(s, t) \leq 3 \operatorname{dist}(s, t)+$ $2 k \operatorname{dist}(s, t) / 2=(k+3) \operatorname{dist}(s, t)<(2 k-1) \operatorname{dist}(s, t)$ for any $k \geq 4$, as required. 
Consider the case where the algorithm returns a value in line 14 . The algorithm returns a value in line 14 in the case where found $2=$ false when the algorithm reaches line 14 . Let $\ell^{\prime}$ be the value of $\ell$ in line 14 of the algorithm. Note that if found $2=$ false in line 14 , then $L_{s}\left[\tilde{d}_{m i n}, \ell^{\prime}-2\right] \geq \tilde{d}$.

Let $i \leftarrow \overline{\operatorname{even}}_{s}\left(L_{s}\left[\tilde{d}_{\text {min }}, \ell\right]\right)$. Notice that $\operatorname{dist}\left(s, p_{i}(s)\right) \geq \operatorname{dist}_{M N}(s, t)$.

We have $\operatorname{dist}(s, t) \geq \Delta_{i}(s) / 2$ and $\operatorname{dist}_{M N}(s, t) \leq \operatorname{dist}\left(s, p_{i}(s)\right) \leq i \Delta_{i}(s) / 2 \leq i \operatorname{dist}(s, t)<$ $k \operatorname{dist}(s, t)<(2 k-1) \operatorname{dist}(s, t)$.

Consider the case where the algorithm returns a value in line 17.

Let $i_{1} \leftarrow \underline{\text { even }}_{s}\left(L_{s}\left[\tilde{d}_{\text {min }}, \ell\right]\right)$. Note that $i^{\prime} \leftarrow \overline{\text { even }}_{s}\left(L_{s}\left[\tilde{d}_{\text {min }}, \ell-1\right]\right)$, satisfies, $i^{\prime}=i_{1}-2$.

In addition, note that in this case Check-Ind $\left(s, t, i_{1}\right) \neq-1$ and Check-Ind $\left(s, t, i_{1}-2\right)=-1$.

Recall that by Lemma 2.1 Check-Ind $\left(s, t, i_{1}\right) \leq 2 \operatorname{dist}\left(s, p_{i_{1}-2}\right)+3 \operatorname{dist}(s, t) \leq 2\left(i_{1}-2\right) \Delta_{i_{1}-2}(s) / 2+$ $\operatorname{dist}(s, t) \leq 2\left(i_{1}-2\right) \leq \operatorname{dist}(s, t)+3 \operatorname{dist}(s, t) \leq(2 k-1) \operatorname{dist}(s, t)$.

We are left with the case where the algorithm returns a value in line 18 . In this case we show that the pair $\left(i_{1}, i_{2}\right)$ returned by the algorithm is a legitimate pair for $s$ and $t$.

1. $i_{2}$ and $i_{1}$ are even by definition of $\overline{\text { even }}_{s}$ and $\underline{\text { even }}_{s}$.

2. As Check-Ind $\left(s, t, i_{1}\right)=-1$ we have $\Delta_{i_{1}}(s) / 2 \leq \operatorname{dist}(s, t)$.

3. As Check-Ind $\left(s, t, i_{2}\right) \neq-1$, we have that either $p_{I\left(s, i_{2}\right)-2}(s) \in B(t)$ or $p_{I\left(s, i_{2}\right)-1}(t) \in B(s)$.

4. Since $\tilde{\mathcal{D}}\left(\operatorname{dist}\left(s, p_{i_{1}}(s)\right)\right)=L_{s}\left[\tilde{d}_{m i n}, \ell^{\prime}\right]$ and $\tilde{\mathcal{D}}\left(\operatorname{dist}\left(s, p_{i_{2}}(s)\right)\right)=L_{s}\left[\tilde{d}_{m i n}, \ell^{\prime}\right]$ then $\operatorname{dist}\left(s, p_{i_{2}}(s)\right) \leq$ $2 \operatorname{dist}\left(s, p_{i_{1}}(s)\right)$.

Lemma 2.3 Procedure Find-Legitimate runs in $O(1)$ time.

Proof: It is easy to verify that all operations in Procedure Find-Legitimate can be done in constant time. We need to show that the number of iterations in each of the while loops is constant.

First not that the number of iterations in the while loop in line 3 of Procedure Find-Legitimate is at most 11 .

Consider now the while loop in line 7 of Procedure Find-Legitimate . Note that $L_{s}\left[\tilde{d}_{c u r r}, 0\right] \leq$ $2 \operatorname{dist}_{M N}(s, t)$ and that $L_{s}\left[\tilde{d}_{c u r r}, j^{\prime}-1\right] \leq L_{s}\left[\tilde{d}_{c u r r}, j^{\prime}\right] / 2$. It is not hard to see now that the number of iteration in the while loop of line 7 is $O(1)$.

Finally, consider the while loop of line 12 . Recall that $\tilde{d}_{\text {min }}$ found in the algorithm is the maximal value in $H_{s}$ such that $\tilde{d}_{\text {min }} \leq \tilde{d} / 256$.

Hence $L_{s}\left[\tilde{d}_{\text {min }}, 9\right] \geq \tilde{d}$. Therefore when $\ell=10$, we have $L_{s}\left[\tilde{d}_{\text {min }}, \ell-2\right] \geq \tilde{d}$. It follows by the condition of the while loop that the number of iterations is at most $O(1)$.

\subsection{Estimate the Distance given Legitimate Pair}

We now present a procedure Estimate that given two legitimate indices $i_{1}$ and $i_{2}$, returns in constant time a distance within stretch $2 k-1$.

The algorithm stores in the preprocessing the following information.

For every node $v$ and every even index $1 \leq i \leq k-1$, store the following.

The minimal even index $x_{1}(i, v)$ such that $1 \leq x_{1}(i, v) \leq k-1$ and $\left(x_{1}(i, v)-i\right)\left(\Delta_{x_{1}(i, v)}(v)-\right.$ $\left.\Delta_{i}(v)\right) \geq\left(k-x_{1}(i, v)-2\right) \Delta_{i}(v)$.

The minimal even index $x_{2}(i, v)$ such that $x_{1}(i, v) \leq x_{2}(i, v) \leq k-1$ and $\left(x_{2}(i, v)-x_{1}(i, v)\right)\left(\Delta_{x_{2}(i, v)}(v)-\right.$ $\left.\Delta_{x_{1}(i, v)}(v)\right) \geq\left(k-x_{2}(i, v)-2\right) \Delta_{x_{1}(i, v)}(v)$. 
The minimal even index $x_{3}(i, v)$ such that $x_{2}(i, v) \leq x_{3}(i, v) \leq k-1$ and $\left(x_{3}(i, v)-x_{2}(i, v)\right)\left(\Delta_{x_{3}(i, v)}(v)-\right.$ $\left.\Delta_{x_{2}(i, v)}(v)\right) \geq\left(x_{1}(i, v)\right)\left(\Delta_{x_{2}(i, v)}(v)-\Delta_{x_{1}(i, v)}(v)\right)$, if no such index exists set $x_{3}(i, v)$ to be the maximum even index (namely, either $k-2$ or $k-1$ ).

Procedure Estimate given $i_{1}, i_{2}$ operates as follows.

Let $x_{1}=x_{1}\left(i_{1}, s\right), x_{2}=x_{2}\left(i_{1}, s\right)$ and $x_{3}=x_{3}\left(i_{1}, s\right)$.

If Check-Ind $\left(s, t, x_{1}\right) \neq-1$ then return Check-Ind $\left(s, t, x_{1}\right)$. Else if Check-Ind $\left(s, t, x_{2}\right) \neq-1$ then return Check-Ind $\left(s, t, x_{2}\right)$. Else if Check-Ind $\left(s, t, x_{3}\right) \neq-1$ then return Check-Ind $\left(s, t, x_{3}\right)$. Else return Check-Ind $\left(s, t, i_{2}\right)$.

We now turn to the analysis of the algorithm.

We first show that $x_{1}(i, v)$ and $x_{2}(i, v)$ are well defined for every index even $1 \leq i \leq k-1$. Note that $x_{3}(i, v)$ is well defined as if there is no index that satisfy the inequality then $x_{3}(i, v)$ is set to be either $k-2$ or $k-1$.

Claim 2.2 For every node $v$ and even index $1 \leq i \leq k-1, x_{1}(i, v), x_{2}(i, v)$ are well defined.

Proof: Let $x_{1}=x_{1}(i, v)$. Recall that $x_{1}$ is the the minimal even index such that $\left(x_{1}-i\right)\left(\Delta_{x_{1}}(v)-\right.$ $\left.\Delta_{i}(v)\right) \geq\left(k-x_{1}-2\right) \Delta_{i}(v)$. We need to show that such an index exists. If $k-1$ is even then note that $x_{1}^{\prime}=k-1$ satisfies the inequality. Similarly, if $k-2$ is even then note that $x_{1}^{\prime}=k-2$ satisfies the inequality. As one of $k-1$ and $k-2$ is even then $x_{1}$ is well defined. Similarly, we can show that $x_{2}(i, v)$ is well defined.

Lemma 2.4 Given a pair of nodes $s$ and $t$ and a legitimate pair $i_{1}$ and $i_{2}$ for $s$ and $t$, Procedure Estimate returns a distance $\hat{d}$ such that $\operatorname{dist}(s, t) \leq \hat{d} \leq(2 k-1) \operatorname{dist}(s, t)$.

Proof: Notice the the algorithm may halt in one of the following lines: (2)-(5). We consider these four cases (corresponding to the different lines in which the algorithm may halt in) and show that the lemma holds in each such case.

The first case is when the algorithm halts in line 2, namely, when Check-Ind $\left(s, t, x_{1}\right) \neq-1$.

By Lemma2.1 either Check-Ind $\left(s, t, x_{1}\right) \leq(2 k-1) \operatorname{dist}(s, t)$ or Check-Ind $\left(s, t, x_{1}\right) \leq 2 \operatorname{dist}\left(s, p_{x_{1}-2}(s)\right)+$ $3 \operatorname{dist}(s, t)$. We need therefore to show that $2 \operatorname{dist}\left(s, p_{x_{1}-2}(s)\right)+3 \operatorname{dist}(s, t) \leq(2 k-1) \operatorname{dist}(s, t)$.

Note that by the minimality of $x_{1}$, we have $\left(x_{1}-2-i_{1}\right)\left(\Delta_{x_{1}-2}(s)-\Delta_{i_{1}}(s)\right)<\left(k-x_{1}+2-2\right) \Delta_{i_{1}}(s)$. Note also that for every even $j \geq i_{1}$ we have, $\operatorname{dist}\left(s, p_{j}(s)\right) \leq i_{1} \Delta_{i_{1}}(s) / 2+\left(j-i_{1}\right) \Delta_{j}(s) / 2$.

It follows that

$$
\begin{aligned}
\hat{d} & \leq 2 \operatorname{dist}\left(s, p_{x_{1}-2}(s)\right)+3 \operatorname{dist}(s, t) \\
& \leq 2 i_{1} \Delta_{i_{1}}(s) / 2+2\left(x_{1}-2-i_{1}\right) \Delta_{x_{1}-2}(s) / 2+3 \operatorname{dist}(s, t) \\
& =i_{1} \Delta_{i_{1}}(s)+\left(x_{1}-2-i_{1}\right) \Delta_{x_{1}-2}(s)+3 \operatorname{dist}(s, t) \\
& \leq i_{1} \Delta_{i_{1}}(s)+\left(x_{1}-2-i_{1}\right)\left(\Delta_{x_{1}-2}(s)-\Delta_{i_{1}}(s)\right)+\left(x_{1}-2-i_{1}\right) \Delta_{i_{1}}(s)+3 \operatorname{dist}(s, t) \\
& =\left(x_{1}-2\right) \Delta_{i_{1}}(s)+\left(x_{1}-2-i_{1}\right)\left(\Delta_{x_{1}-2}(s)-\Delta_{i_{1}}(s)\right)+3 \operatorname{dist}(s, t) \\
& \leq\left(x_{1}-2\right) \Delta_{i_{1}}(s)+\left(k-x_{1}+2-2\right) \Delta_{i_{1}}(s)+3 \operatorname{dist}(s, t) \\
& =(k-2) \Delta_{i_{1}}(s)+3 \operatorname{dist}(s, t) \leq(2 k-4) \operatorname{dist}(s, t)+3 \operatorname{dist}(s, t) \\
& =(2 k-1) \operatorname{dist}(s, t) .
\end{aligned}
$$

Note that if the algorithm did not return a value in line 2, namely, if Check-Ind $\left(s, t, x_{1}\right)=-1$ then by Lemma 2.1, we have $\operatorname{dist}(s, t) \geq \Delta_{x_{1}}(s) / 2$. 
The proof of the case where the algorithm returns a value in step 3 is similar to the previous case (with replacing $x_{1}$ by $x_{2}$ and $i_{1}$ by $x_{1}$ ).

We now turn the the case where the algorithm halts in line 4.

In this case we have by Lemma 2.1, $\operatorname{dist}(s, t) \geq \Delta_{x_{2}}(s) / 2$.

Recall that $x_{3}$ is the minimal even index such that $\left(x_{3}-x_{2}\right)\left(\Delta_{x_{3}}(s)-\Delta_{x_{2}}(s)\right) \geq\left(x_{1}\right)\left(\Delta_{x_{2}}(s)-\right.$ $\Delta_{x_{1}}(s)$ ), or the maximum even index if no such index exists.

By the minimality of $x_{3}$, we have $\left(x_{3}-2-x_{2}\right)\left(\Delta_{x_{3}-2}(s)-\Delta_{x_{2}}(s)\right)<\left(x_{1}\right)\left(\Delta_{x_{2}}(s)-\Delta_{x_{1}}(s)\right)$. Therefore $\left(x_{3}-2-x_{2}\right)\left(\Delta_{x_{3}-2}(s)\right)<\left(x_{1}\right)\left(\Delta_{x_{2}}(s)-\Delta_{x_{1}}(s)\right)+\left(x_{3}-2-x_{2}\right) \Delta_{x_{2}}(s)$. Note that, $\operatorname{dist}\left(s, p_{x_{3}-2}(s)\right) \leq x_{1} \cdot \Delta_{x_{1}}(s) / 2+\left(x_{2}-x_{1}\right) \Delta_{x_{2}}(s) / 2+\left(x_{3}-2-x_{2}\right) \Delta_{x_{3}-2}(s) / 2$.

We get that,

$$
\begin{aligned}
\operatorname{dist}\left(s, p_{x_{3}-2}(s)\right) & \leq x_{1} \Delta_{x_{1}}(s) / 2+\left(x_{2}-x_{1}\right) \Delta_{x_{2}}(s) / 2+\left(x_{3}-2-x_{2}\right) \Delta_{x_{3}-2}(s) / 2 \\
& \leq x_{1} \Delta_{x_{1}}(s) / 2+\left(x_{2}-x_{1}\right) \Delta_{x_{2}}(s) / 2+\left(x_{1}\right)\left(\Delta_{x_{2}}(s)-\Delta_{x_{1}}(s)\right) / 2+\left(x_{3}-2-x_{2} \Delta_{x_{2}}(s)\right) / 2 \\
& =\left(x_{3}-2\right) \Delta_{x_{2}}(s) / 2
\end{aligned}
$$

Hence $\hat{d}=2 \operatorname{dist}\left(s, p_{x_{3}-2}(s)\right)+3 \operatorname{dist}(s, t) \leq\left(x_{3}-2\right) \Delta_{x_{2}}(s)+3 \operatorname{dist}(s, t) \leq 2\left(x_{3}-2\right) \operatorname{dist}(s, t)+$ $3 \operatorname{dist}(s, t)=\left(2 x_{3}-1\right) \operatorname{dist}(s, t) \leq(2 k-1) \operatorname{dist}(s, t)$.

Finally, consider the case where the algorithm returns a value in line 5 . Note that in this case, we have $\operatorname{dist}(s, t) \geq \Delta_{x_{3}}(s) / 2$. In addition note that $x_{3}$ is not the maximal even index as otherwise Check-Ind $\left(s, t, x_{3}\right) \neq-1$. Hence by definition $x_{3}$ satisfies $\left(x_{3}-x_{2}\right)\left(\Delta_{x_{3}}(s)-\Delta_{x_{2}}(s)\right) \geq$ $\left(x_{1}\right)\left(\Delta_{x_{2}}(s)-\Delta_{x_{1}}(s)\right)$.

We claim that in this case $\Delta_{x_{3}}(s) \geq 2 \Delta_{i_{1}}(s)$.

To show this we consider two cases, the first is when $x_{1} \leq\left(k+i_{1}-2\right) / 2$ and the second when $x_{1}>\left(k+i_{1}-2\right) / 2$.

Consider the first case where $x_{1} \leq\left(k+i_{1}-2\right) / 2$. We have, $\left(\Delta_{x_{1}}(s)-\Delta_{i_{1}}(s)\right) \geq\left(k-x_{1}-\right.$ $2) \Delta_{i}(s) /\left(x_{1}-i_{1}\right) \geq \Delta_{i_{1}}(s) \cdot\left(k-\left(k+i_{1}-2\right) / 2-2\right) /\left(\left(k+i_{1}-2\right) / 2-i_{1}\right)=\Delta_{i_{1}}(s)$. We get that $\Delta_{x_{1}}(s) \geq 2 \Delta_{i_{1}}(s)$. Hence $\Delta_{x_{3}}(s) \geq \Delta_{x_{1}}(s) \geq 2 \Delta_{i_{1}}(s)$.

Consider now the second case where $x_{1}>\left(k+i_{1}-2\right) / 2$.

$$
\begin{aligned}
\left(\Delta_{x_{3}}(s)-\Delta_{x_{2}}(s)\right) & \geq\left(x_{1}\right)\left(\Delta_{x_{2}}(s)-\Delta_{x_{1}}(s)\right) /\left(x_{3}-x_{2}\right) \\
& \geq\left(x_{1}\right)\left(k-x_{2}-2\right) \Delta_{x_{1}}(s) /\left(x_{3}-x_{2}\right)\left(x_{2}-x_{1}\right) \\
& \geq\left(x_{1}\right)\left(x_{3}-x_{2}\right) \Delta_{x_{1}}(s) /\left(x_{3}-x_{2}\right)\left(x_{2}-x_{1}\right) \\
& =\left(x_{1}\right) \Delta_{x_{1}}(s) /\left(x_{2}-x_{1}\right) \\
& >\Delta_{x_{1}}(s)\left(k+i_{1}-2\right) / 2\left(x_{2}-\left(k+i_{1}-2\right) / 2\right) \\
& =\Delta_{x_{1}}(s)\left(k+i_{1}-2\right) /\left(2 x_{2}-\left(k+i_{1}-2\right)\right) \\
& \geq \Delta_{x_{1}}(s)\left(k+i_{1}-2\right) /\left(2(k-2)-\left(k+i_{1}-2\right)\right) \\
& =\Delta_{x_{1}}(s)\left(k+i_{1}-2\right) /\left(k-2-i_{1}\right) \\
& \geq \Delta_{x_{1}}(s) .
\end{aligned}
$$

We get that, $\Delta_{x_{3}}(s) \geq \Delta_{x_{2}}(s)+\Delta_{x_{1}}(s) \geq 2 \Delta_{i_{1}}(s)$ as required. 
We thus have

$$
\begin{aligned}
\operatorname{dist}(s, t) \leq \hat{d} & \leq 2 \operatorname{dist}\left(s, p_{I\left(i_{2}, s\right)-2}(s)\right)+3 \operatorname{dist}(s, t) \\
& \leq 2 \operatorname{dist}\left(s, p_{i_{2}}(s)\right)-2 \Delta_{i_{2}}(s)+3 \operatorname{dist}(s, t) \\
& \leq 2 \operatorname{dist}\left(s, p_{i_{2}}(s)\right)-\operatorname{dist}(s, t) \\
& \leq 4 \operatorname{dist}\left(s, p_{i_{1}}(s)\right)-\operatorname{dist}(s, t) \\
& \leq 2 i_{1} \Delta_{i_{1}}(s)-\operatorname{dist}(s, t) \\
& \leq 2 i_{1} \operatorname{dist}(s, t)-\operatorname{dist}(s, t) \\
& \leq 2(k-1) \operatorname{dist}(s, t)-\operatorname{dist}(s, t) \\
& <(2 k-1) \operatorname{dist}(s, t) .
\end{aligned}
$$

\subsection{Running time and space}

The analysis of the running time and space is similar to the one presented in [16] and is brought here for completeness.

The Mendel-Naor distance oracle 9] can be constructed in $O\left(n^{2+1 / k} \log n\right)$ time and requires $O\left(n^{1+1 / k}\right)$ time.

Similar to the construction of Wulff-Nilsen [16], our construction can use any distance oracle with $O(c \cdot k)$ stretch and $O(1)$ time that can output at most $O\left(n^{1+1 / k}\right)$ different distances, for any constant $c$. More precisely, our query algorithm can be slightly modified such that given a distance oracle with $O(c \cdot k)$ stretch and $O(1)$ time, the query time is $O(\log c)$ for some integer $c$. This can be done by modifying Procedure Find-Legitimate and having $O(\log c)$ iterations in the while loops. Constructing such a distance oracle with $O(c k)$ stretch and $O(1)$ query time can be done using the construction of Mendel and Schwob [10] in $O\left(m n^{1 / c k} \log ^{3} n\right)$.

Constructing the bunches and the pivots can be done by the Thorup-Zwick [14] analysis in $O\left(k m n^{1 / k}\right)$.

As shown in [16], the set $\mathcal{D}_{M N}$ contains $O\left(n^{1+1 / k}\right)$ values. In addition, the set $\mathcal{D}_{T Z}$ contains $O\left(k n^{1+1 / k}\right)$ values. Hence the set $\mathcal{D}$ contains $O\left(k n^{1+1 / k}\right)$ values. Sorting the values in $\mathcal{D}$ and forming the set $\tilde{\mathcal{D}}$ can be done in $O\left(k n^{1+1 / k} \log n\right)$ time.

Finding the values $\tilde{\mathcal{D}}(d)$ for every distance $d \in \mathcal{D}$ can be done in $|\mathcal{D}|$ by traversing in parallel both lists $\tilde{\mathcal{D}}$ and $\mathcal{D}$ in increasing order as follows. Initially, set $j, i=0$. While not reaching the end of both lists do the following. If $\mathcal{D}[j] \leq \tilde{\mathcal{D}}[i]$, set $\tilde{\mathcal{D}}(d)=\tilde{\mathcal{D}}[i]$, where $d=\mathcal{D}[j]$ and set $j \leftarrow j+1$. Else $(\mathcal{D}[j]>\tilde{\mathcal{D}}[i])$, set $i \leftarrow i+1$. It is not hard to see that this process takes $O(|\mathcal{D}|)$ time.

Consider a node $u$. It is not hard to see that finding the values $\delta_{j}(u)$ for every index $1 \leq j \leq k-1$ can be done in $O(k)$ time by simply calculating $\delta_{j}(u)=\operatorname{dist}\left(u, p_{j}(u)\right)-\operatorname{dist}\left(u, p_{j-2}(u)\right)$.

Finding the values $\Delta_{j}(u)$ for every index $1 \leq j \leq k-1$ can be done in $O(k \log k)$ time by the following. First sort the values $\delta_{j}(u)$ (this takes $O(k \log k)$ time). Next, sequentially traverse the indices $i$ from 1 to $k$ and maintaining the largest value $\delta_{j}(u)$ observed so far. Constructing the hash $h_{u}$ and $L_{u}$ can also be done in time $O(k)$.

Finally calculating the values $x_{1}(i, u), x_{2}(i, u), x_{3}(i, u)$ can be done in time $O\left(k^{2}\right)$ as follows.

For every pair of indices $i, j$ find $(j-i)\left(\Delta_{j}(v)-\Delta_{i}(v)\right)$ and $(k-j-2) \Delta_{i}(v)$ 
Set $x_{1}(i, v)$ to be the minimal even index such that $1 \leq x_{1}(i, v) \leq k-1$ and $\left(x_{1}(i, v)-\right.$ $i)\left(\Delta_{x_{1}(i, v)}(v)-\Delta_{i}(v)\right) \geq\left(k-x_{1}(i, v)-2\right) \Delta_{i}(v)$. This can be done by simply exhaustive search on all indices.

Similarly set $x_{2}(i, v)$ to be the minimal even index such that $x_{2}(i, v) \leq k-1$ and $\left(x_{2}(i, v)-\right.$ $\left.x_{1}(i, v)\right)\left(\Delta_{x_{2}(i, v)}(v)-\Delta_{x_{1}(i, v)}(v)\right) \geq\left(k-x_{2}(i, v)-2\right) \Delta_{x_{1}(i, v)}(v)$.

Similarity set $x_{3}(i, v)$ to be the minimal even index such that $x_{3}(i, v) \leq k-1$ and $\left(x_{3}(i, v)-\right.$ $\left.x_{2}(i, v)\right)\left(\Delta_{x_{3}(i, v)}(v)-\Delta_{x_{2}(i, v)}(v)\right) \geq\left(x_{1}(i, v)\right)\left(\Delta_{x_{2}(i, v)}(v)-\Delta_{x_{1}(i, v)}(v)\right)$, if no such index exists set $x_{3}(i, v)$ to be the maximum even index (namely, either $k-2$ or $k-1$ ).

We get that the preprocessing time for each node $u$ is $O\left(k^{2}\right) \leq O(k \log n)$ and thus for all nodes $O(n k \log n)$ time.

All in all the preprocessing time for given integers $k$ and $c$ is $O\left(k m n^{1 / k}+k n^{1+1 / k} \log n+\right.$ $\left.m n 1 /(c k) \log ^{3} n\right)$.

In addition, it is not hard to verify that the size of the data structure is $O\left(k n^{1+1 / k}\right)$.

\section{References}

[1] I. Abraham, and C. Gavoille. On Approximate Distance Labels and Routing Schemes with Affine Stretch. DISC, 404-415, 2011.

[2] B. Awerbuch, B. Berger, L. Cowen, and D. Peleg. Near-linear time construction of sparse neighborhood covers. In SIAM J. Comput., Vol. 28, No. 1, 263-277, 1998.

[3] S. Baswana, A. Gaur, S. Sen and J. Upadhyay. Distance oracles for unwieghted graphs: Breaking the quadratic barrier with constant additive error. In ICALP, pages 609-621, 2008.

[4] S. Baswana and T. Kavitha. Faster algorithms for approximate distance oracles and all-pairs small stretch paths. In Proc. IEEE Symp. on Foundations of Computer Science (FOCS), 591-602, 2006.

[5] E. Cohen. Fast algorithms for constructing t-spanners and paths with stretch t. SIAM J. Comput., 28:210-236, 1998.

[6] P. Erdős. Extremal problems in graph theory. In Theory of graphs and its applications, pages 29-36, 1964.

[7] J. Matoušek. On the distortion required for embeding finite metric spaces into normed spaces. In Israel Journal of Math 93, 333-344, 1996.

[8] M. Mendel, and A. Naor. Ramsey partitions and proximity data structures In Proc. 47th IEEE Symp. on Foundations of Computer Science (FOCS), 109-118, 2006.

[9] M. Mendel, and A. Naor. Ramsey partitions and proximity data structures. In Journal of the European Mathematical Society, 9:2, 253-275, 2007.

[10] M. Mendel and C. Schwob. Fast C-K-R Partitions of Sparse Graphs. In Journal of Theoretical Comp. Sci., (2), 1-18, 2009.

[11] A. Naor, and T. Tao. Scale-oblivious metric fragmentation and the nonlinear Dvoretzky theorem. In Israel Journal of Mathematics, 192 ,489-504, 2012.

[12] M. Pătraşcu and L. Roditty. Distance oracles beyond the thorup-zwick bound. In FOCS, pages 815-823, 2010.

[13] L. Roditty, M. Thorup, and U. Zwick. Deterministic constructions of approximate distance oracles and spanners. In Proc. 32nd Int. Colloq. on Automata, Languages 83 Prog., 261-272, 2005.

[14] M. Thorup and U. Zwick. Approximate distance oracles. In J. ACM, 52, 1-24, 2005. 
[15] C. Wulff-Nilsen. Approximate Distance Oracles with Improved Preprocessing Time. In Proc. 23rd ACM-SIAM Symposium on Discrete Algorithms (SODA),202-208, 2012.

[16] C. Wulff-Nilsen. Approximate Distance Oracles with Improved Query Time. In Proc. 24th ACM-SIAM Symp. on Discrete Algorithms (SODA), 539-549, 2013. 
1. Let $\tilde{d}=\tilde{\mathcal{D}}\left(\operatorname{dist}_{M N}(s, t)\right)$.

2. If $s \in B(t)$ or $t \in B(s)$ then return $(-1,-1$, $\operatorname{dist}(s, t))$.

3. Set $i=2$ and found $1 \leftarrow$ false.

4. While $(i>-9)$ and $($ found $1=$ false $)$ do:

(a) Let $\tilde{d}_{\text {curr }}=\tilde{\mathcal{D}}[\tilde{d}, i]$.

(b) If $H_{s}\left(\tilde{d}_{\text {curr }}\right) \neq$ null or $H_{t}\left(\tilde{d}_{\text {curr }}\right) \neq$ null then found $1=$ true.

(c) Else $i=i-1$.

5. If found $1=$ false then return $\left(-1,-1,2 \tilde{d}_{\text {curr }}\right)$.

6. If $H_{s}\left(\tilde{d}_{\text {curr }}\right)=$ null then set $(s, t) \leftarrow(t, s)$.

7. Set $j=0$.

8. While $\left(L_{s}\left[\tilde{d}_{c u r r}, j\right]>\operatorname{dist}_{M N}(s, t) / 256\right)$

(a) $j \leftarrow j-1$.

9. $\tilde{d}_{\text {min }} \leftarrow L_{s}\left[\tilde{d}_{c u r r}, j\right]$.

10. Let $i_{\text {min }} \leftarrow \overline{\text { even }}_{s}\left(\tilde{d}_{\text {min }}\right)$.

11. If Check-Ind $\left(s, t, i_{\text {min }}\right) \neq-1$, then $\operatorname{return}\left(-1,-1\right.$, Check-Ind $\left.\left(s, t, i_{\text {min }}\right)\right)$.

12. $\ell \leftarrow 0$, found $2 \leftarrow$ false

13. While $($ found $2=$ false $)$ and $\left(L_{s}\left[\tilde{d}_{\text {min }}, \ell-2\right]<\tilde{d}\right)$

(a) Let $i \leftarrow \overline{\operatorname{even}}_{s}\left(L_{s}\left[\tilde{d}_{\text {min }}, \ell\right]\right)$.

(b) $d=$ Check-Ind $(s, t, i)$.

(c) If $d \neq-1$ then found $\leftarrow$ true else $\ell \leftarrow \ell+1$.

14. If found $2=$ false then return $\left(-1,-1\right.$, $\left.\operatorname{dist}_{M N}(s, t)\right)$.

15. $i_{2} \leftarrow I\left(s, \overline{\operatorname{even}}_{s}\left(L_{s}\left[\tilde{d}_{m i n}, \ell\right]\right)\right)$.

16. $i_{1} \leftarrow \underline{\text { even }}_{s}\left(L_{s}\left[\tilde{d}_{m i n}, \ell\right]\right)$.

17. If Check-Ind $\left(s, t, i_{1}\right) \neq-1$, then return $\left(-1,-1\right.$, Check-Ind $\left.\left(s, t, i_{1}\right)\right)$.

18. Else return $\left(i_{1}, i_{2},-1\right)$.

Figure 1: Estimate the distance 
algorithm $\operatorname{Estimate}\left(G, i_{1}, i_{2}\right)$

1. Let $x_{1}=x_{1}\left(i_{1}, s\right), x_{2}=x_{2}\left(i_{1}, s\right)$ and $x_{3}=x_{3}\left(i_{1}, s\right)$.

2. If Check-Ind $\left(s, t, x_{1}\right) \neq-1$ then return Check-Ind $\left(s, t, x_{1}\right)$.

3. If Check-Ind $\left(s, t, x_{2}\right) \neq-1$ then return Check-Ind $\left(s, t, x_{2}\right)$.

4. If Check-Ind $\left(s, t, x_{3}\right) \neq-1$ then return Check-Ind $\left(s, t, x_{3}\right)$.

5. Else return Check-Ind $\left(s, t, i_{2}\right)$.

Figure 2: Estimate the distance 